\title{
Optimal number of endoscopic biopsies for diagnosis of early gastric cancer
}

\section{(ㄷ)(요 $\odot$}

\author{
Authors \\ Hisashi Doyama ${ }^{1}$ \\ Institutions \\ 1 Department of Gastroenterology, Ishikawa Prefectural \\ Central Hospital, Kanazawa, Japan \\ 2 Department of Diagnostic Pathology, Ishikawa \\ Prefectural Central Hospital, Kanazawa, Japan \\ 3 Department of Gastroenterology, Kanazawa University \\ Hospital, Kanazawa, Japan
}

Masaki Nishitani ${ }^{1,3}$, Naohiro Yoshida ${ }^{1}$, Shigetsugu Tsuji ${ }^{1}$, Teppei Masunaga ${ }^{1}$, Hirokazu Hirai ${ }^{1}$, Saori Miyajima ${ }^{1}$, Akihiro Dejima ${ }^{1}$, Takashi Nakashima ${ }^{1}$, Shigenori Wakita', Kenichi Takemura ${ }^{1}$, Hiroshi Minato², Shuichi Kaneko ${ }^{3}$,

submitted 30.4.2019

accepted after revision 5.8.2019

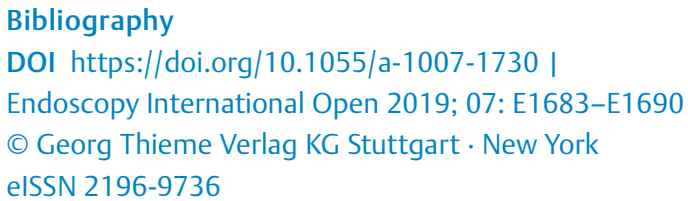

Corresponding author

Naohiro Yoshida, Department of Gastroenterology, Ishikawa Prefectural Central Hospital, 2-1 Kuratsukihigashi, Kanazawa, Ishikawa 920-8530, Japan

Fax: +81-76-238-2337

ynaohiro@ipch.jp

\section{ABSTRACT}

Background and study aims No recommendations are available for optimal number of endoscopic biopsies for early gastric cancer (GC), and whether detection of early $\mathrm{GC}$ is improved by increasing the number of biopsy is unclear. We therefore evaluated the relationship between number of biopsies and diagnostic accuracy.

Materials and methods We retrospectively evaluated 858 early GCs ( 623 from endoscopic submucosal dissection and 235 surgical specimens), which we classified as obtained after one, two, or three or more biopsies. We assessed diagnostic accuracy by number of biopsies, and in subgroups by tumor diameter, gross type, and surface color.

Results Almost half the lesions were obtained after one biopsy each, $30 \%$ after two biopsies, and $20 \%$ after three or more biopsies. Although diagnostic accuracy increased with biopsy number, it was significantly greater for the two-biopsy group than the one-biopsy group, (92.5\% vs. $83.9 \%, P=0.0009)$, but did not significantly differ between the two- and three or more-biopsy groups. This finding was seen when tumors were evaluated by size, but not by elevated type and surface color, for which more biopsies did not improve diagnostic accuracy. Multivariate analysis demonstrated that two or more biopsies was the independent significant factors for diagnostic accuracy.

Conclusions Two biopsies are the optimal number required to diagnose early GC.

\section{Introduction}

Gastric cancer (GC) is the third most common cause of cancerrelated fatalities world-wide [1]. As with other cancers, early diagnosis is an important factor in surviving GC, and commonly requires endoscopic screening [2].

Endoscopy-based GC diagnosis often uses magnifying endoscopy with narrow-band imaging (M-NBI) [3-5], for which the vessel plus surface classification system was established and used as an "optical biopsy" [4, 6]. However, pathological diagnosis using biopsy specimens is still the gold standard for diagnosing GC.
Although multiple biopsies have been recommended for diagnosis of advanced GC $[7,8]$, almost no recommendations or guidelines are available for diagnosis of early GC. In the clinical setting, we perform multiple biopsies from detected lesions, but whether diagnostic accuracy for early GC is improved with more biopsies is unclear. We therefore evaluated the relationship between number of biopsy specimens and diagnostic accuracy to find the optimal number of biopsies for early GC. 


\section{Patients and methods}

\section{Study design and objectives}

This retrospective study was conducted at the Ishikawa Prefectural Central Hospital, a tertiary referral center in Japan. In accordance with the Declaration of Helsinki, the institutional review board of Ishikawa Prefectural Central Hospital approved this study.

Early GCs that were treated by endoscopic submucosal dissection (ESD) or surgery in our hospital were included in this study. Exclusion criteria were as follows: 1) histological diagnosis made in other hospitals; 2) details of biopsy unknown (e.g. the order of biopsies); and 3) intentional biopsy from only the surrounding non-cancerous mucosa (i.e. mapping biopsy). To investigate the optimal number of biopsies for correctly diagnosing early GC, we assessed diagnostic accuracy of endoscopic biopsy according to number of specimens taken from the one lesion.

\section{Definition of GC and demographic characteristics}

Preoperative histological diagnoses were based on Group Classification according to the Japanese Classification of Gastric Carcinoma (JCGC) [9]: Group 4 (neoplastic lesion that is suspected to be carcinoma) and Group 5 (carcinoma) were preoperatively diagnosed as GC. Group 1 (normal tissue or non-neoplastic lesion), Group 2 (material for which diagnosis of neoplastic or non-neoplastic lesion is difficult), and Group 3 (adenoma) were diagnosed as not GC.

Specimens postoperatively diagnosed as GC were histologically typed according to the JCGC [9]. Malignant epithelial tumors, including papillary, tubular (well and moderately differentiated), poorly differentiated (solid and non-solid type), signet-ring cell, and mucinous, were diagnosed as GC. Mixed adenocarcinoma (e.g., por2 > sig > tub2) was also diagnosed as GC.

Tumors confined to the mucosa or submucosa (irrespective of lymph node metastasis) were considered early GC. Tumor diameter was maximum size; we divided lesions into $\geq 10 \mathrm{~mm}$ and $<10 \mathrm{~mm}$. We defined GCs $<5 \mathrm{~mm}$ in the longest diameter as minute gastric cancer (mGC), and those measuring 5 to $10 \mathrm{~mm}$ in the longest diameter as small gastric cancer (sGC). Gross types were classified as 0 -I (protruding), 0-Ila (superficial elevated), 0-Ilb (superficial flat), 0-Ilc (superficial depressed), and 0-III (excavated), according to the JCGC [9]. These were reclassified as two groups in this study: 0 -I and/or 0 -Ila were considered elevated type, and the remaining types and lesions with more than 2 components except for 0 -I and Ila were classified as non-elevated type. Color of the lesion was classified into reddened/same color, pale color, or uncertain. In this study, we analyzed reddened/same and pale.

All pathological diagnosis was performed by two expert pathologists, with all results double-checked.

\section{Endoscopic system and procedure}

We used a single company's endoscope, a video processor, and a light source (Olympus Co., Tokyo Japan). The NBI system became available in 2007 . In our routine practice, we examined the entire stomach with conventional white-light imaging
(C-WLI) to detect suspicious lesions for GC, which had irregular borders, irregular surfaces, and/or non-homogeneous color. When we detected such lesions, we performed detailed examination by chromoendoscopy and/or M-NBI. Then we performed biopsies from the cancerous mucosa.

\section{Endoscopists}

Endoscopic examinations were carried out by experts and trainees. Expert endoscopists had at least 6 years' experience in endoscopy with WLI and M-NBI. Trainees had less than 6 years' experience in endoscopy. In our hospital, trainees must perform diagnostic endoscopies and biopsies alongside experts. Therefore, all examinations were performed by experts only or by experts and trainees.

\section{Endpoints}

The primary endpoint was difference in diagnostic accuracy of endoscopic biopsy according to number of biopsy specimens taken from one lesion. Diagnostic accuracy was defined as a sensitivity of endoscopic biopsy for diagnosing GC: proportion of GC that was accurately diagnosed by endoscopic biopsy among histologically proven GC in resected specimen. When any of biopsy specimen from one lesion was cancerous, the lesion was diagnosed as GC and judged as an accurate diagnosis. On the other hand, when all biopsy specimens from one lesion were Group 1, 2, and 3, the lesion was diagnosed as non-GC and judged as an incorrect diagnosis. In patients who were received multiple preoperative endoscopies and biopsies for the same lesion in our hospital, the result of the last endoscopy was used for analysis.

Secondary endpoints were contributions of tumor diameter, gross type and surface color to diagnostic accuracy.

\section{Statistical analysis}

Continuous variables were reported as medians and percentages with $95 \%$ confidence intervals (Cls). Categorical variables were compared using the $\mathrm{X} 2$ or Fisher's exact test when the expected values were less than five. Multivariate logistic regression analysis regarding the factors for preoperatively diagnosed as GC or not GC was performed to identify independence of clinical factors. $P<0.05$ was considered significant. All statistical analyses were performed with EZR (version 1.36, Saitama Medical Center, Jichi Medical University, Saitama, Japan), which is a graphical user interface for R (The R Foundation for Statistical Computing, Vienna, Austria). More precisely, it is a modified version of $\mathrm{R}$ commander designed to add statistical functions frequently used in biostatistics [10].

\section{Results}

From January 2004 to July 2016, 2528 early GCs were treated by ESD or surgery in our hospital. We excluded 1632 lesions because they were biopsied in other hospitals, and 38 lesions because we could not ascertain their pathological diagnoses for each biopsy specimen. Thus, a total of 858 lesions were analyzed (623 from ESD, 235 from surgeries; - Fig. 1). Adenocarci- 
Lesions assessed for enrollment $\mathbf{n}=\mathbf{2 5 2 8}$

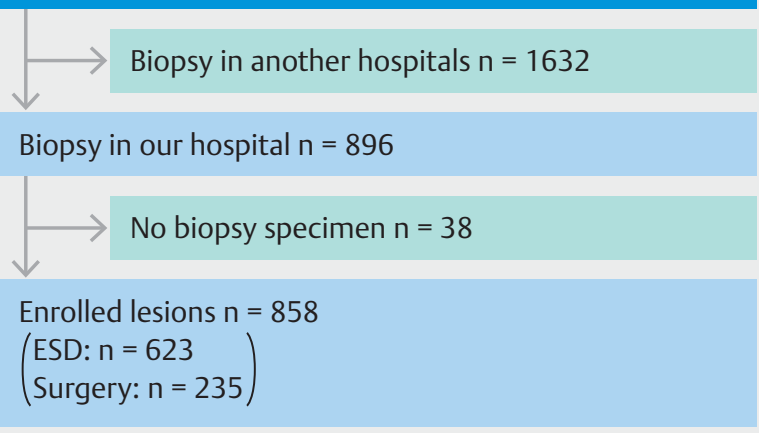

ESD: endoscopic submucosal dissection

Fig. 1 Flow diagram of patients enrolled in the current study.

noma was the postoperative diagnosis for all lesions; highgrade adenoma was not included.

Clinicopathological characteristics of 858 lesions are summarized in $>$ Table 1 . Almost half had one biopsy, $30 \%$ lesions had two biopsies, and $20 \%$ had three or more biopsies. All lesions had biopsies diagnosed as Groups 2 to 5 . In preoperative biopsies, 762 lesions (89\%) were Group 4 or Group 5, 75 lesions (9\%) were Group 3, and 21 lesions (2\%) were Group 2.

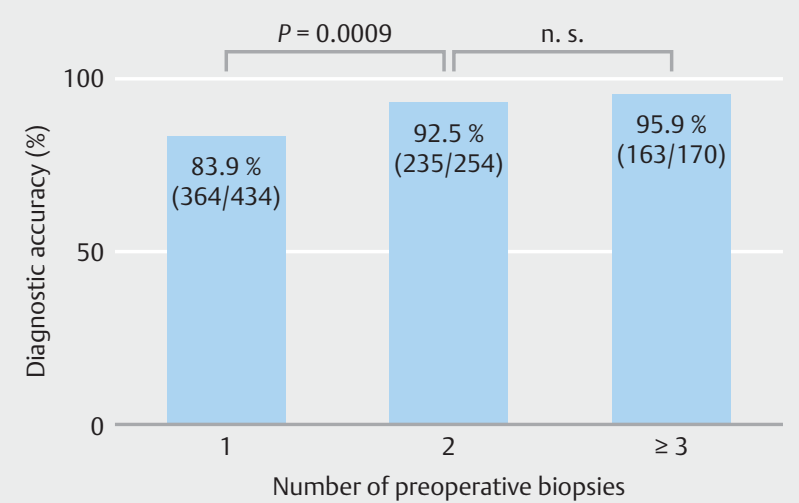

n. s.: not significant

- Fig. 2 Diagnostic accuracy according to number of biopsy specimens in 858 early gastric cancer lesions.

Diagnostic accuracy increased with number of biopsy specimens ( $\triangleright$ Fig. 2). It was $92.5 \%$ (95\% Cl: $88.6-95.4 \%)$ when at least two specimens were taken, which was significantly higher than in the one-biopsy group (83.9\% [95\% Cl: $80.1-87.2 \%$ ]; $P=$ 0.0009). However, although diagnostic accuracy was $95.9 \%$ (95\% Cl: $91.7-98.3 \%$ ) in the three or more-biopsy group, it was not significantly higher than in the two-biopsy group.

Diagnostic accuracy for both the $\geq 10 \mathrm{~mm}$ and $<10 \mathrm{~mm}$ tumor diameter subgroups also increased with number of biop-

- Table 1 Clinicopathological characteristics of 858 lesions according to the number of biopsy specimens.

\begin{tabular}{|c|c|c|c|}
\hline & \multicolumn{3}{|c|}{ Number of preoperative biopsies } \\
\hline & $1(n=434)$ & $2(n=254)$ & $\geq 3(n=170)$ \\
\hline \multicolumn{4}{|c|}{ Biopsy histology classification, $n$ (\%) (highest Group in biopsy specimens) } \\
\hline - Group 2 & $17(4.0)$ & $4(1.6)$ & $0(0.0)$ \\
\hline - Group 3 & $53(12.2)$ & $15(5.9)$ & $7(4.2)$ \\
\hline - Group 4 & $83(19.1)$ & $32(12.6)$ & $5(2.9)$ \\
\hline - Group 5 & $281(64.7)$ & 203 (79.9) & $158(92.9)$ \\
\hline Tumor median diameter, mm (range) & $12(2-122)$ & $18(1-123)$ & $30.5(1-230)$ \\
\hline \multicolumn{4}{|l|}{ Tumor diameter, $n(\%)$} \\
\hline . $<10 \mathrm{~mm}$ & $164(37.8)$ & $47(18.5)$ & $11(6.5)$ \\
\hline - $\geq 10 \mathrm{~mm}$ & $270(62.2)$ & $207(81.5)$ & $159(93.5)$ \\
\hline \multicolumn{4}{|l|}{ Gross type, $n(\%)$} \\
\hline - Elevated & $150(34.6)$ & $60(23.6)$ & $26(15.3)$ \\
\hline - Non-elevated & $284(65.4)$ & $194(76.4)$ & $144(84.7)$ \\
\hline \multicolumn{4}{|l|}{ Surface color, n (\%) } \\
\hline - Reddened/same & $316(72.8)$ & $166(65.4)$ & $68(40.0)$ \\
\hline - Pale & $107(24.7)$ & $44(17.3)$ & $34(20.0)$ \\
\hline - Uncertain & $11(2.5)$ & $44(17.3)$ & $68(40.0)$ \\
\hline
\end{tabular}




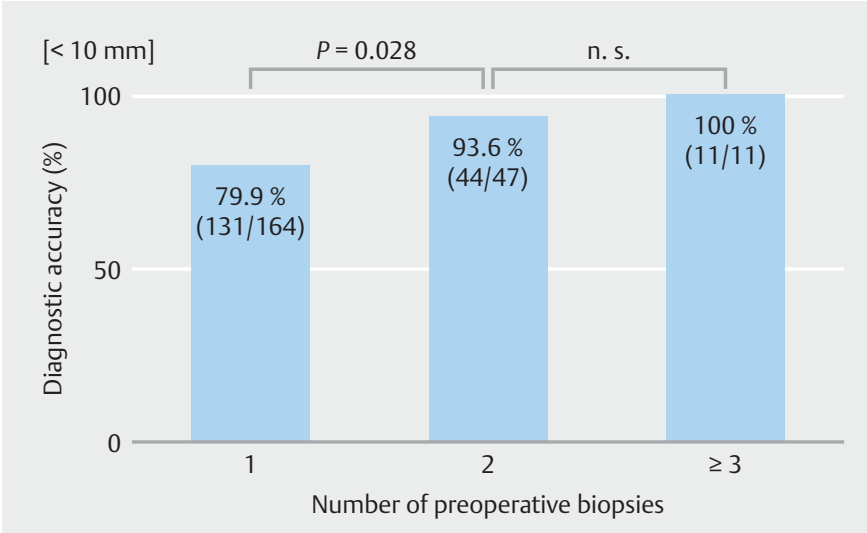

n. s.: not significant

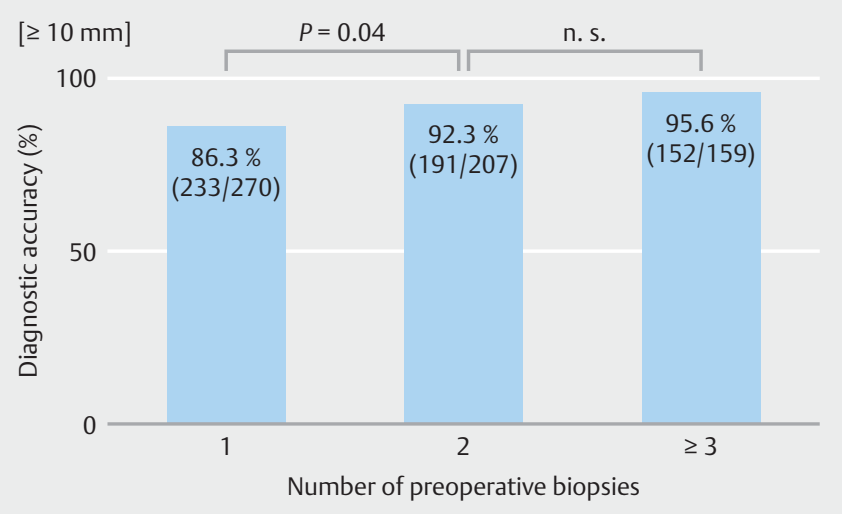

n. s.: not significant

Fig. 3 Diagnostic accuracy according to number of biopsy specimens, classified by tumor diameter ( $<10 \mathrm{~mm}$ or $\geq 10 \mathrm{~mm})$.

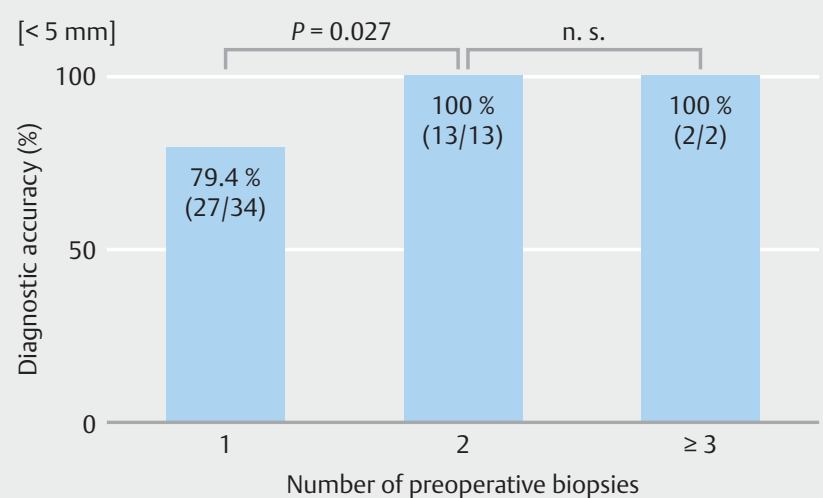

n. s.: not significant

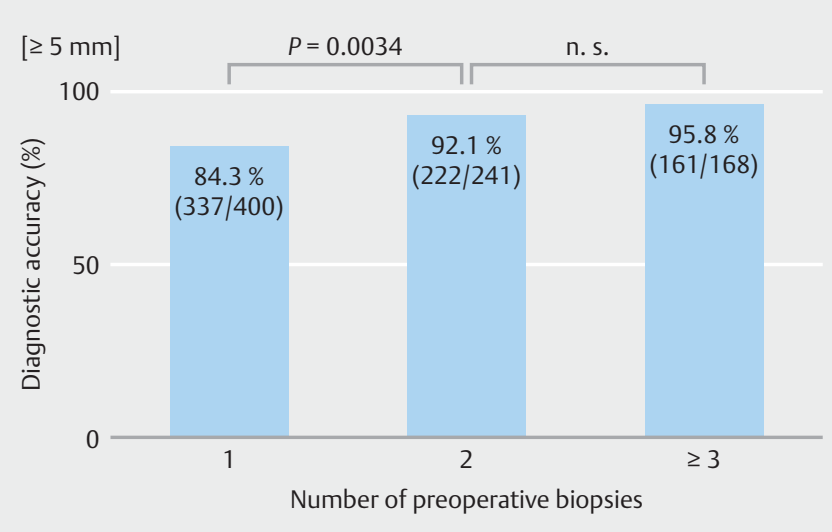

n. s.: not significant

Fig. 4 Diagnostic accuracy according to number of biopsy specimens, classified by tumor diameter ( $<5 \mathrm{~mm}$ or $\geq 5 \mathrm{~mm}$ ).

sies ( $\boldsymbol{\text { Fig. }} \mathbf{3}$ ). Irrespective of tumor size, diagnostic accuracy increased over $90 \%$ when two specimens were collected, and was significantly higher than when only one specimen was collected (<10 mm: $79.9 \%[95 \% \mathrm{Cl}: 72.9-85.7 \%]$ to $93.6 \%[95 \%$ Cl: $82.5-98.7 \%$ ], $P=0.028, \geq 10 \mathrm{~mm}: 86.3 \%$ [95\% Cl: $81.6-$ $90.2 \%$ ] to $92.3 \%$ [ $95 \% \mathrm{Cl}: 87.8-95.5 \%$ ], $P=0.04$ ), but did not significantly differ between the two- and three or more-biopsy groups.

In this connection, we also assessed the contribution of tumor diameter ( $\geq 5 \mathrm{~mm}$ vs. $<5 \mathrm{~mm}$ ) to diagnostic accuracy. Similarly, diagnostic accuracy was significantly improved by increasing biopsy specimens from one to two ( $\mathbf{F i g . 4}$ ).

Diagnostic accuracy by gross type (including both elevated type and non-elevated type) also increased with number of biopsies ( $\mathbf{F i g . 5}$ ) - significantly improved between one and two biopsies for the non-elevated type, but not for the elevated type (from about $70 \%$ to $80 \%$ ).

Diagnostic accuracy by surface color (including both reddened/same color and pale color) tended to increase with number of biopsies, but not significantly so ( $\mathbf{F i g . 6}$ ). In the pale color, diagnostic accuracy was from about $70 \%$ to $80 \%$.
- Table 2 compares factors associated with preoperative histological diagnosis about GC or not GC. Univariate analysis, these factors, except for surface color: reddened/same, were significantly positively associated with preoperative diagnosis of GC. Multivariate analysis identified two factors - number of preoperative biopsies two or more and gross type: non-elevated - as independent significant predictors for GC.

\section{Discussion}

Our results show that diagnostic accuracy significantly increased as biopsy specimens went from one to two, but not between two and three or more biopsies. In addition to this, performing two or more biopsies was the independent significant factor for diagnosis of early GC. Therefore, the optimal number of biopsies required to diagnose early GC is apparently two. There are two possible explanations for why two biopsies are better than one. The first is that two biopsies would reduce sampling error, especially for small lesions. The second is that two specimens may raise pathologists' confidence level of diagnosing GC more than one specimen. 


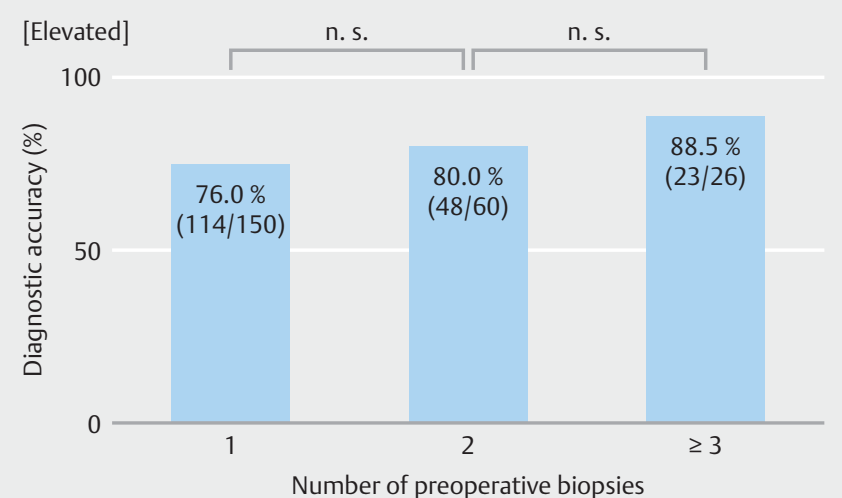

n. s.: not significant

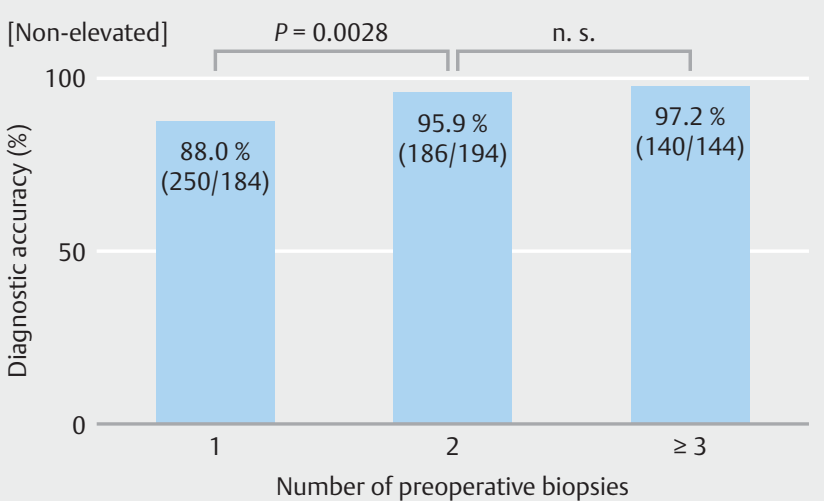

n. s.: not significant

- Fig. 5 Diagnostic accuracy according to number of biopsy specimens, classified by gross type (elevated or non-elevated).

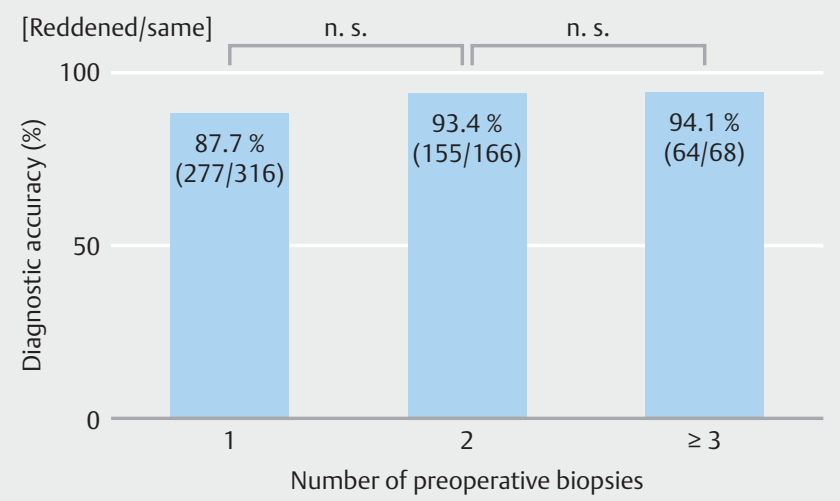

n. s.: not significant

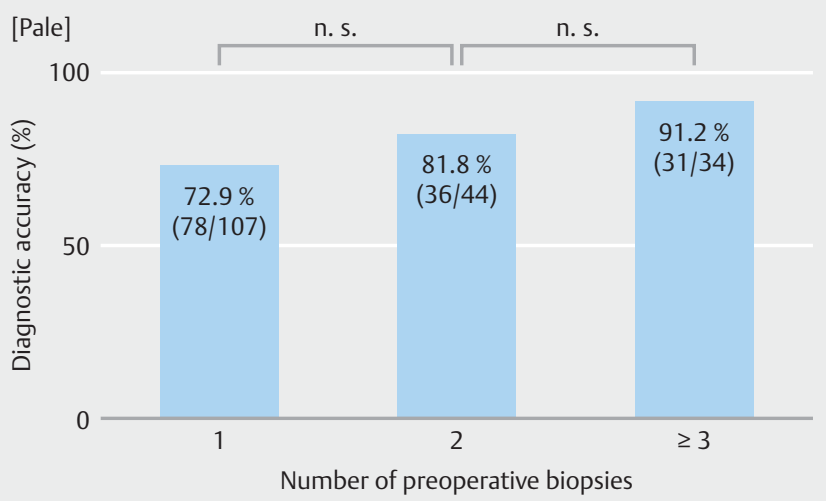

n. s.: not significant

- Fig.6 Diagnostic accuracy according to number of biopsy specimens, classified by surface color (reddened/same or pale).

Multiple biopsies are considered to increase diagnostic accuracy of GC. Several studies published in the $1970 \mathrm{~s}$ and $1980 \mathrm{~s}$ showed that the diagnostic accuracy for GC could reach $97.4 \%$ to $100 \%$ after five or more biopsies of suspected lesions [1114]. Guidelines based on these studies led to recommendations for eight biopsies in Germany, six in UK and six to eight biopsies in the United States to diagnose GC [15-17]. However, these guidelines were mainly intended to diagnose advanced GC, not early GC. Although many studies concluded that antithrombotics did not increase risk of bleeding $[18,19]$, biopsy can cause local fibrosis, which may make endoscopic treatment difficult. As most early GC now can be effectively treated by endoscopy [20], this might become a major problem. Greater numbers of biopsies also increase the workloads of pathologists. Optimization of the number of biopsies for diagnosis of early GC can solve these problems. We therefore performed this study, which indicated that the optimal number of biopsies is two.

In this study, lesions that were preoperatively diagnosed as Group 2 or 3 were considered to be incorrectly diagnosed. Because this study had many more Group 3 lesions than Group 2 lesions, Group 3 was considered to have influenced our results. We often preoperatively perceive lesions to be gastric adenomas that are revealed to be well-differentiated adenocarcinomas postoperatively. In the report, which analyzed efficacy of endoscopic mucosal resection for Group 3 lesions, 37.2\% were found histopathologically to be adenocarcinoma [21]. Preoperatively distinguishing adenocarcinoma from adenoma by biopsy can be challenging. Misdiagnoses of biopsies may be caused by: (a) biopsy specimens that are taken only from the adenoma section though the lesion has both of GC and adenoma components; and (b) small biopsy samples might be inadequate for accurate diagnosis [22]. Also, some misdiagnoses may be due to inadequate biopsies which are diagnostically or technically unable to taken from within cancer. Endoscopic evaluations - including findings of redness, nodularity, and depression by C-WLI - are reportedly useful in differentiating between adenoma and GC [23]. In recent years, combining M-NBI and C-WLI has been reported to enable more accurate differential diagnoses $[4,5,22]$. In contrast to biopsies, which are often obtained from a single point of a lesion, a major advantage of endoscopic evaluations is the ability to assess an entire lesion at 
- Table 2 Univariate and multivariate logistic regression analysis of factors for preoperatively diagnosed as GC or not GC.

\begin{tabular}{|c|c|c|c|c|c|c|}
\hline & \multicolumn{2}{|c|}{ Preoperative histological diagnosis } & \multicolumn{2}{|l|}{ Univariate analysis } & \multicolumn{2}{|c|}{ Multivariate analysis } \\
\hline & GC $(n=762)$ & Not GC $(n=96)$ & OR $(95 \% \mathrm{CI})$ & $P$ value & OR $(95 \% \mathrm{CI})$ & $P$ value \\
\hline $\begin{array}{l}\text { Number of preoperative } \\
\text { biopsies: } \geq 2\end{array}$ & & & $2.94(1.84-4.72)$ & $<0.0001$ & $2.38(1.44-3.94)$ & 0.0007 \\
\hline$(+)$ & 398 & 26 & & & & \\
\hline$(-)$ & 364 & 70 & & & & \\
\hline Tumor diameter: $\geq 10 \mathrm{~mm}$ & & & $1.86(1.19-2.90)$ & 0.0063 & $1.59(0.98-2.56)$ & 0.059 \\
\hline$(+)$ & 576 & 60 & & & & \\
\hline$(-)$ & 186 & 36 & & & & \\
\hline Gross type: Non-elevated & & & $3.53(2.29-5.45)$ & $<0.0001$ & $2.95(1.87-4.66)$ & $<0.0001$ \\
\hline$(+)$ & 577 & 45 & & & & \\
\hline$(-)$ & 185 & 51 & & & & \\
\hline Surface color: Reddened/same & & & $1.38(0.90-2.13)$ & 0.14 & $2.26(0.89-2.26)$ & 0.146 \\
\hline$(+)$ & 495 & 55 & & & & \\
\hline$(-)$ & 267 & 41 & & & & \\
\hline
\end{tabular}

once. Targeted biopsies from the area where the cancer is exposed, guided by endoscopic findings, may reduce preoperative misdiagnoses. However, biopsy samples that are too small for accurate diagnosis are a remaining problem. Although increasing the number of biopsies is one way to address this problem, comprehensive endoscopic and histopathological diagnosis would probably be a better strategy, considering the high utility of endoscopy, and the problems of biopsy (i.e., treating lesions that are strongly suspected to be GC by endoscopic evaluation, even if they are later diagnosed as Group 3 by biopsy). Progress with the technique of endoscopic resection also supports this strategy. When lesions are removed endoscopically, we can obtain a large specimen, which enables more precise histological diagnosis compared to biopsy. Therefore, we suggest that two biopsies are optimal, and that a treatment plan should be determined by comprehensive endoscopic and histopathological diagnosis.

In this study, irrespective of tumor size, diagnostic accuracy significantly increased with increasing biopsy specimens from one to two. Some studies have classified small GC lesions into $\mathrm{mGC}$ and $\mathrm{SGC}$, and reported that such small lesions were difficult to diagnose and detect $[23,24]$. Obviously, we should try to diagnose cancer accurately while it is as small as possible, because such small cancers can be cured by endoscopic surgery. Our study suggests performing two biopsies, even from mGC or sGC, for accurate diagnosis. However, two biopsies could cause separation or disappearance of very small lesions, making them difficult to detect during endoscopic resection. Therefore, these lesions should be treated as soon as possible, before scars of the biopsies disappear.

For elevated, reddened, and same or pale lesions, diagnostic accuracy did not significantly increase when we increased the number of biopsies from one to two. When limited to elevated type and pale color, diagnostic accuracy was only 70 to $80 \%$. This result might reflect the percentage of adenoma (elevated type: $21.2 \%$; non-elevated type: $4.0 \%$; reddened/same: $5.7 \%$; pale: $19.4 \%$ ). For these types of lesions, comprehensive diagnosis, including endoscopic evaluation, may be more important.

Our study has several limitations. First, as it was a retrospective study, we could calculate only sensitivity as a diagnostic performance; therefore, prospective studies are desired. Second, we could not completely exclude effect of information bias; Large lesions might be performed multiple biopsies, and small lesions might be performed one biopsy. Third, backgrounds of the three groups differed in ways that could not be adjusted by subset analyses. Fourth, the endoscopic system varied over the period of this study. Although use of $\mathrm{M}-\mathrm{NBI}$ might influence the result, it was impossible to precisely extract only the lesion which was diagnosed by M-NBI. For reference, we divided the lesions into two groups with 2008 when $\mathrm{M}-\mathrm{NBI}$ became stable technique, and we confirmed that there was no statistical difference of diagnostic accuracy between two groups ( $>$ Supplementary Fig.).

\section{Conclusion}

In conclusion, our findings suggest that two biopsies are the optimal number needed to diagnose early GC. Comprehensive endoscopic and histopathological diagnoses are important in the clinical setting. 


\section{Acknowledgements}

The authors thank Marla Brunker from Edanz Group (www. edanzediting.com/ac) for editing a draft of this manuscript

\section{Competing interests}

None

\section{References}

[1] Fitzmaurice C, Akinyemiju TF, Al Lami FH et al. Global, regional, and national cancer incidence, mortality, years of life lost, years lived with disability, and disability-adjusted life-years for 29 cancer groups, 1990 to 2016. JAMA Oncol 2018; 4: $1553-1568$

[2] Jun JK, Choi KS, Lee HY et al. Effectiveness of the Korean National Cancer Screening Program in reducing gastric cancer mortality. Gastroenterology 2017; 152: 1319-1928

[3] Muto M, Katada C, Sano Y et al. Narrow band imaging: a new diagnostic approach to visualize angiogenesis in superficial neoplasia. Clin Gastroenterol Hepatol 2005; 3: 16-20

[4] Yao K, Anagnostopoulos GK, Ragunath K. Magnifying endoscopy for diagnosing and delineating early gastric cancer. Endoscopy 2009; 41: $462-467$

[5] Ezoe $\mathrm{Y}$, Muto $\mathrm{M}$, Uedo $\mathrm{N}$ et al. Magnifying narrowband imaging is more accurate than conventional white-light imaging in diagnosis of gastric mucosal cancer. Gastroenterology 2011; 141: 2017 - 2025

[6] Yao K, Doyama H, Gotoda T et al. Diagnostic performance and limitations of magnifying narrow-band imaging in screening endoscopy of early gastric cancer: a prospective multicenter feasibility study. Gastric Cancer 2014; 17: 669-679

[7] Hale MD, Gotoda T, Hayden JD et al. Endoscopic biopsies from gastrointestinal carcinomas and their suitability for molecular analysis: a review of the literature and recommendations for clinical practice and research. Histopathology 2015; 67: 147 - 157

[8] Choi Y, Choi HS, Jeon WK et al. Optimal number of endoscopic biopsies in diagnosis of advanced gastric and colorectal cancer. J Korean Med Sci 2012; 27: 36 - 39

[9] JGCA. Japanese classification of gastric carcinoma: 3rd English edition. Gastric Cancer 2011; 14: 101-112
[10] Kanda Y. Investigation of the freely-available easy-to-use software "EZR" (Easy R) for medical statistics. Bone Marrow Transplant 2013; 48: $452-458$

[11] Dekker W, Tytgat GN. Diagnostic accuracy of fiberendoscopy in the detection of upper intestinal malignancy. A follow-up analysis. Gastroenterology 1977; 73: $710-714$

[12] Misumi A, Mori K, Ikeda T et al. Evaluation of fibergastroscopic biopsy in the diagnosis of gastric cancer: a study of 339 cases. Gastroenterol. Jpn 1978; 13: 255-263

[13] Sancho-Poch FJ, Balanzó J, Ocaña J et al. An evaluation of gastric biopsy in the diagnosis of gastric cancer. Gastrointest. Endosc 1978; 24: $281-282$

[14] Tatsuta M, lishi H, Okuda S et al. Prospective evaluation of diagnostic accuracy of gastrofiberscopic biopsy in diagnosis of gastric cancer. Cancer 1989; 63: 1415-1420

[15] Moehler M, Al-Batran SE, Andus T et al. German S3-guideline "Diagnosis and treatment of esophagogastric cancer". Z Gastroenterol 2011; 49: $461-531$

[16] Allum WH, Blazeby JM, Griffin SM. Guidelines for the management of oesophageal and gastric cancer. Gut 2011; 60: 1449-1472

[17] Ajani JA, Bentrem DJ, Besh S et al. Gastric cancer, version 2. 2013: featured updates to the NCCN Guidelines. J Natl Compr. Canc Netw 2013; 11: $531-546$

[18] Sieg A, Hachmoeller-Eisenbach U, Eisenbach T. Prospective evaluation of complications in outpatient $\mathrm{Gl}$ endoscopy: a survey among German gastroenterologists. Gastrointest Endosc 2001; 53: 620-627

[19] Johnson B, Basson MD. Absence of complications after endoscopic mucosal biopsy. Dig. Dis 2018; 36: 328-332

[20] Ono H, Yao K, Fujishiro M et al. Guidelines for endoscopic submucosal dissection and endoscopic mucosal resection for early gastric cancer. Dig. Endosc 2016; 28: 3-15

[21] Katsube T, Konnno S, Hamaguchi K et al. The efficacy of endoscopic mucosal resection in the diagnosis and treatment of group III gastric lesions. Anticancer Res 2005; 25: 3513-3516

[22] Miwa K, Doyama H, Ito R et al. Can magnifying endoscopy with narrow band imaging be useful for low grade adenomas in preoperative biopsy specimens? Gastric Cancer 2012; 15: $170-178$

[23] Kozawa H, Nakazawa S, Yoshino J. Clinical and pathological study on minute and small gastric cancers, with special reference to their macroscopic classification. Gastroenterol Endosc 1985; 27 : $1523-$ 1537

[24] Takahashi H, Hirata K, Sawada S et al. Endoscopic diagnosis of minute gastric cancers. Gastroenterol Endosc 2011; 53: 1229-1240 


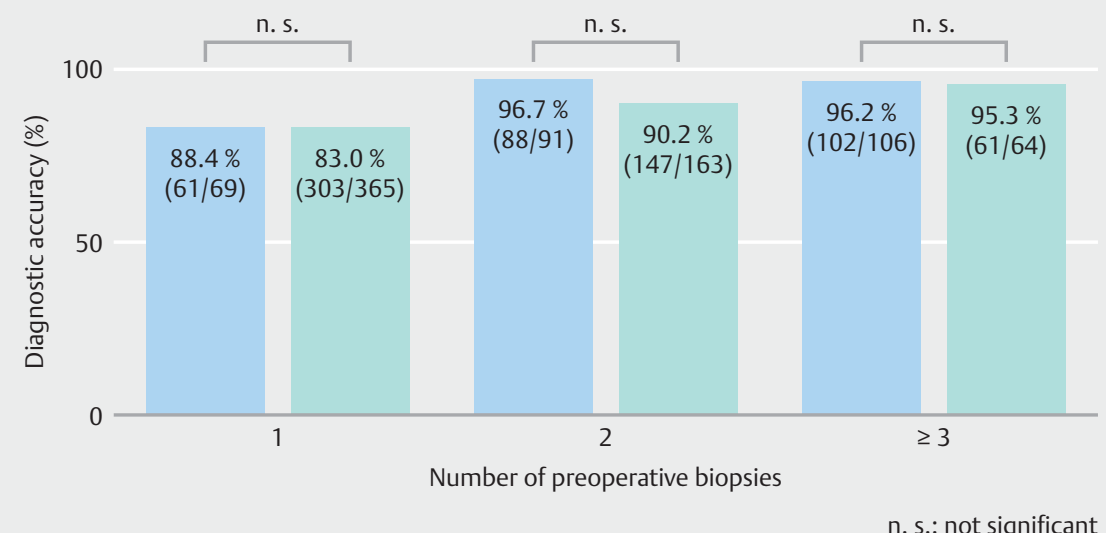

- Supplementary Fig. Diagnostic accuracy according to number of biopsy specimens, divided into two groups with 2008 (the "blue" bar and the "green" bar show " $<2008$ " and " $\geq 2008$," respectively). 